\title{
How students describe infinitesimal sources and infinitesimal spaces in integrals
}

\author{
Gregory Mulder, Paul J. Emigh, and Elizabeth Gire
}

Department of Physics, Oregon State University, 301 Weniger Hall, Corvallis, OR 97330

\begin{abstract}
Many students who have completed a full year of calculus continue to demonstrate a weak understanding of the role of the infinitesimal in an integral. This lack of understanding has implications for learning in introductory calculus-based physics, where students must integrate contributions from spatially-distributed sources. Symbolically, a student who constructs such an integral in terms of small chunks of sources, like $d m$ or $d q$, will need to relate their infinitesimal to a small chunk of space, like $d x$. In order to better understand how students use the integration infinitesimal, we collected and analyzed written solutions from 39 upperdivision physics majors who solved for the electric field at an asymmetric point in space above a homogeneously charged bar. We have identified several categories of ways students handled the infinitesimal when constructing an integral. We present evidence, supporting prior work, that a small segment understanding of the infinitesimal leads students to a correct translation from $d q$ to $d x$.
\end{abstract}

\section{INTRODUCTION}

In introductory calculus-based physics, students are commonly asked to solve problems that require converting an infinitesimal from a physical source quantity to a spatial quantity. For example, a student might be asked to convert $d m$ to $d x$ when using the generalized coordinate-independent center-of-mass equation:

$$
x_{c m}=\frac{1}{M} \int x d m
$$

For example, in Knight's Physics with Calculus for Scientists and Engineers [1], students are introduced to the concept of linear mass density in order to construct a conversion of $d m$ to $d x$ via the relationship:

$$
d m=\frac{M}{L} d x
$$

Here, the concept of linear mass density facilitates conversion from an infinitesimal source to an infinitesimal space.

In this paper, we address the research question: How do middle-division physics majors communicate their use of the infinitesimal in written format? We present results from students who were asked to find the electric field due to a bar of charge at a non-symmetric point above the bar.

We first discuss some prior research that has examined students' use of and conceptualizations about infinitesimals. Then we present results from 39 junior-level physics majors who were asked to solve a particular problem in an electrostatics course. We conclude with some implications our results may have for teaching physics at both the introductory and upper-division level.

\section{PRIOR RESEARCH}

$\mathrm{Hu}$ and Rebello [2] conducted teaching/learning interviews with 13 engineering students enrolled in the $2^{\text {nd }}$ semester of introductory calculus-based physics. In this study, they identified three resources that students used involving infinitesimals, or, as $\mathrm{Hu}$ calls them, differentials:
- The "Small piece/segment" resource refers to small piece of a physical object. With at least one group, activation of this resource led to a successful $d q$ to $d x$ relationship.

- The "Point" resource refers to a conceptualization of the infinitesimals $d x$ and $d q$ as point quantities. Although this resource was based in a physical understanding of the concept of point charges within a very small space, activation of this resource led at least one group of students to describe $d q$ as equal to $Q / L$ (incorrectly).

- The "Differentiation" resource sees the " $d$ " as a mathematical operator with little or no physical meaning. Activation of this resource led students down a path they could not explain "other than mathematically."

Additionally, Jones [3] conducted interviews with eight university students and used Sherin's Symbolic Forms [4] to analyze the productivity of different schematic conceptualizations of the integral. Each student was first asked "mathematics-framed" tasks that made use of integrals similar to those found in calculus textbooks. A week later, the same students were asked "physics-framed" tasks similar to those found in calculus-based physics and engineering textbooks.

Jones then discussed the relative productivity of three conceptions that he described as:

- "Function Matching" is similar to Hu's "Differentiation". In this conceptualization students tried to play a matching game where integrands come from some other "original function" which became the integrand via a derivative. Jones reported that function mapping does not seem to help people solve physics problems

- In "Perimeter and Area" reasoning, students view the integral limits and the integrand as the defining characteristics of the region of which the integral is attempting to find the area.

- "Adding up Pieces" aligns closely with Hu's "Small piece/segment" resource with Jones claiming that this conception leads to the best physics understanding. 
A non-conducting rod has a length $L$ and a total charge $Q$ distributed uniformly along its length.

What is the electric field at a point a distance $d$ above one end of the charged rod, as shown below?

Note: the electric field from a point charge is: $\vec{E}=\frac{1}{4 \pi \varepsilon_{o}} \frac{q}{r^{2}} \hat{r}$

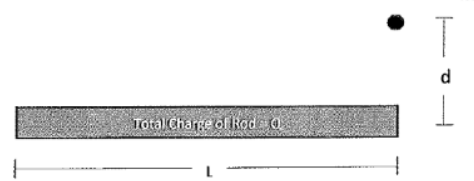

FIG 1. A "Refresher" question given to physics majors at the beginning of the Junior year.

In our presentation below, our findings build upon claims made by both $\mathrm{Hu}$ and Jones that understanding the infinitesimal as a small segment is more likely to lead to correct problem solving.

\section{METHODS AND ANALYSIS}

Our research was conducted with 39 students enrolled in a middle-division electrostatics course at Oregon State University. Most of these students were physics majors. As with many large state universities, the students may have completed their introductory calculus-based physics sequence in-house, at another institution or, in some cases, as part of a high school AP course. In the first week of their Junior year, we asked these students to solve the "Refresher" question shown in Fig. 1. Students worked independently for about 20 minutes. Digital and paper copies were made for later analysis.

The data was analyzed paying attention to how student acts of coordinatization in their problem solving. In this case coordinatization refers to methods of applying a coordinative system to a coordinate-independent equation. In the case of electrostatics, the electric field from a point charge is often represented as:

$$
\vec{E}=\frac{1}{4 \pi \varepsilon_{0}} \frac{q}{r^{2}} \hat{r}
$$

When applying this generalized coordinate-independent equation to find the electric field from a distribution of charges, problem solvers must draw upon resources gained from a variety of math and physics classes.

The analysis began with the first author performing an open-coding [5] to identify common acts of coordinatization across the data set. These individual acts were then grouped into broad categories corresponding to patterns that we observed in the data. The authors then discussed the codes $f$

TABLE I. Category codes developed to describe student acts of employing a coordinate system while solving the "Refresher" question. ( $\mathrm{N}=39$ )

\begin{tabular}{l|ll}
\hline \multicolumn{1}{c|}{ Category Code } & N & \% \\
\hline Drawing Axis & 20 & $51 \%$ \\
Manipulating an Infinitesimal & 35 & $90 \%$ \\
Indicates an Infinitesimal in a Sketch & 25 & $64 \%$ \\
Dealing with the Vector Nature of $E$ & 32 & $82 \%$ \\
Indicating Limits of Integration & 34 & $87 \%$ \\
\hline
\end{tabular}

or individual acts of coordinatization as well as the categories, and successive passes were made at the data to refine the analysis.

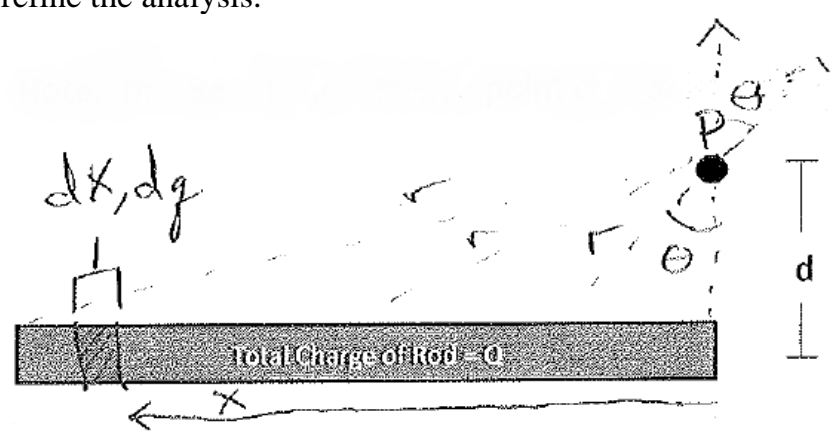

FIG 2. An example of a student indicating both a spatial infinitesimal $(d x)$ and an infinitesimal source $(d q)$.

\section{RESULTS}

We find that students performed a diversity of acts of coordinatization across five categories (see Table I). In this paper, we focus our attention on the two categories related to the infinitesimal: "Manipulating an Infinitesimal" and "Indicating an Infinitesimal in a Sketch." Each of these two categories is described in more detail below, along with examples of student work. Then, we describe an interesting intersection between the two categories that emerged from examining the co-occurrence of the categories: representing an infinitesimal graphically was correlated with use of the correct relationship between the source infinitesimal $d q$ and the spatial infinitesimal $d x$.

\section{A. Indicating an Infinitesimal in a Sketch}

We found that 25 out of 39 students drew or labeled a pictorial representation of the infinitesimal. In some sketches, a small segment of the bar of charge was denoted or labeled with $d q$ (or $d Q$ ). In others, students identified an infinitesimal element of space, such as $d x, d y$, or even $d \theta$. For example, one student (see Fig. 2) labeled both $d q$ (an infinitesimal source charge) and $d x$ (an infinitesimal element of space) on the same graph. Also note in Fig. 2 that the student explicitly marks a section of the rod and shades it to indicate the small piece of the rod that corresponds to the $d x$ and $d q$.

A less clear indication of an infinitesimal can be seen in Fig. 3. This student drew multiple lines between different points on the rod and the point of interest. They then labeled each line with $r$, likely corresponding to the denominator of the integrand. Note that this student did not mark the rod in a way that directly suggests small pieces, but that the lines labeled $r$ matches what is seen in Fig. 2. We included such examples in the "Indicating an Infinitesimal in a Sketch" category so that it would be inclusive of all possible representations that might correspond to underlying reasoning that is based in infinitesimals or small pieces. 


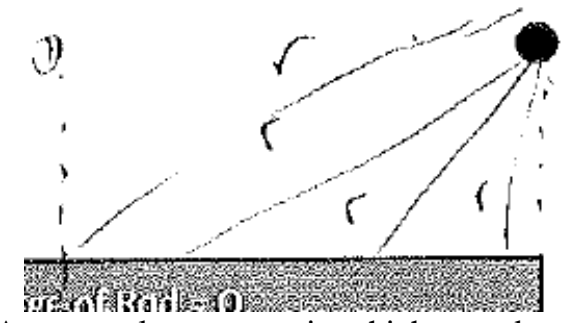

FIG 3. An example response in which a student drew lines to different points along the rod but did not explicitly indicate an infinitesimal.

\section{B. Manipulating an Infinitesimal}

Nearly all students (35 of 39) manipulated an infinitesimal in the symbolic expressions that they wrote. We classified a wide variety of individual acts of coordinatization in this category, summarized in Table II. In this section, we give a brief description of each act.

"Conversion from $d q$ to $\lambda d x$ (correct)" refers to students who wrote a correct conversion from a source infinitesimal to spatial infinitesimal. Many students used $(Q / L)$ instead of $\lambda$. Some students correctly used $d l$ or $d y$ for the spatial infinitesimal, where $l$ or $y$ denote the horizontal axis (see, for example, Fig. 5).

In contrast, some students made a similar but incorrect conversion from a source infinitesimal $(d q)$ to a spatial infinitesimal $(d x)$, omitting any indication of the charge density $\lambda$. This category is labeled "Conversion from $d q$ to $d x$ (incorrect)" in Table II.

"Non-horizontal infinitesimal (e.g., $d r, d y)$ " includes students who used a spatial infinitesimal other than $d x$, where they were specifically not referring to the horizontal direction. (As discussed above, some students used $d l$ or $d y$ to refer to the horizontal direction.) Some students specifically appeared to be integrating in the vertical direction. Others wrote an integral with $d r$, where $r$ likely refers to the $r$ in Coulomb's Law, as shown in Fig. 3. Neither of these infinitesimals is correct because they do not vary along the length of the bar.

Responses were coded as "Source-like infinitesimal" when a student wrote an equation that included either $d E$ or $d q$. Each of these infinitesimals corresponds to a small piece of something that is not space, and either can be

TABLE II. Sub-categories developed to describe student actions within the "Manipulating the Infinitesimal" category. $(\mathrm{N}=39)$

\begin{tabular}{l|ll}
\hline \multicolumn{1}{c|}{ Sub-Category Code } & $\mathbf{N}$ & $\mathbf{\%}$ \\
\hline Conversion from $d q$ to $\lambda d x$ (correct) & 12 & $31 \%$ \\
Conversion from $d q$ to $d x$ (incorrect) & 8 & $21 \%$ \\
Non-horizontal infinitesimal $(e . g ., d r, d y)$ & 7 & $18 \%$ \\
Source-like infinitesimal $(e . g ., d E, d q)$ & 16 & $41 \%$ \\
Double infinitesimal $(e . g ., d x d y, d q d x)$ & 2 & $5 \%$ \\
Infinitesimal in the denominator & 4 & $10 \%$ \\
Description of an infinitesimal in words & 5 & $13 \%$ \\
\hline
\end{tabular}
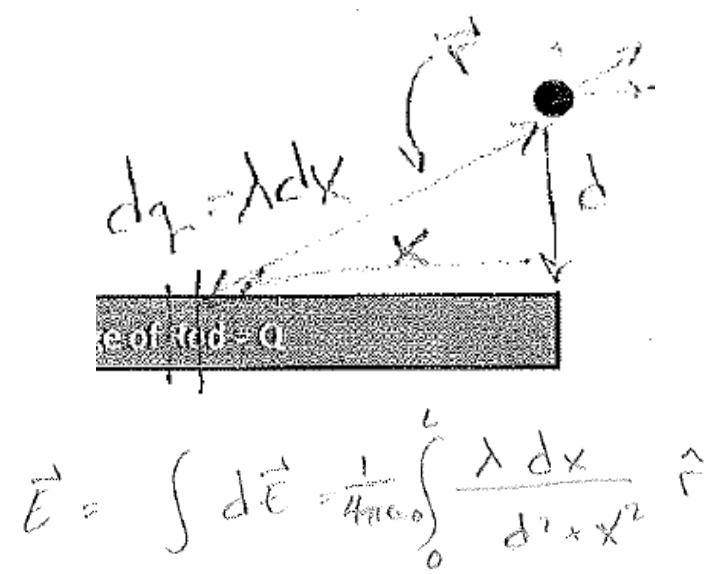

FIG 4. An example of a student that correctly translated the source infinitesimal $d \boldsymbol{E}$ to the spatial infinitesimal $\lambda d x$ accompanying a sketch that includes an infinitesimal.

implemented correctly. In fact, it is not atypical for introductory or upper-division physics textbooks and courses to use $d E$ or $d q$ as intermediate steps in solving electrostatics problems $[1,6]$. Only one of the 16 responses in this subcategory included both $d q$ and $d \boldsymbol{E}$.

A few other actions were relatively infrequent. Two students ("Double infinitesimal") wrote two infinitesimals and two integral signs. Four students ("Infinitesimal in the denominator") wrote an infinitesimal in the denominator inside the integral. Five students ("Description of an infinitesimal in words") indicated an infinitesimal using words either in conjunction with or instead of symbols. While these actions are extremely interesting, we do not focus on them in this article. We intend to investigate student reasoning for these cases in future work.

\section{Co-occurrence of Indicating and Manipulating Infinitesimals}

Given the large number of categories and sub-categories that we identified, we chose to examine possible cooccurrences between the different acts of coordinatization involving infinitesimals. In particular, we focused on two of the more common manipulation actions: "Conversion from $d q$ to $\lambda d x$ (correct)" and "Conversion from $d q$ to $d x$ (incorrect)." We then observed whether or not the students in each of these two categories indicated an infinitesimal in a sketch.

Of the 12 students who correctly used $\lambda$ or $Q / L$ in their manipulation of the infinitesimal, a full $75 \%$ of them also included an infinitesimal in their sketch. One example is shown in Fig. 4. In this example, the student's sketch (top of Fig. 4) includes both a symbolic expression relating infinitesimals, $d q=\lambda d x$, and a pair of marks on the given figure indicating a small piece of the rod. Their symbolic work (bottom of Fig. 4), includes the right-hand side of this expression inside the integral. 

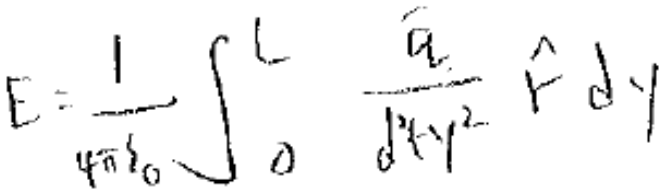

FIG 5. This student did not clearly denote an infinitesimal in their sketch. The only invocation of an infinitesimal is the dy shown here. In this student's sketch, however, the horizontal axis is clearly defined as the $y$-axis.

Meanwhile, for the eight students who had an incorrect infinitesimal, such as $q d x$, appear in their symbolic work, only three $(38 \%)$ drew an infinitesimal in their sketch. In fact, many of the students who had an incorrect or incomplete symbolic implementation of $d x$ displayed no pictorial representation of an infinitesimal and often just jumped into equations, as with the example in Fig. 5. In many of these cases, students just added an integral sign and an infinitesimal sandwiched around the general equation for the E-field from a point charge.

These quantitative results seem to align with the contentions of both $\mathrm{Hu}$ and Rubello [2] and Jones [3] that students drawing upon a "small segment" view of the infinitesimal tend to be more productive in solving physics problems that involve integrals.

\section{CONCLUSIONS}

This research expands on prior work and suggests that, like introductory students more generally, a significant number of middle-division physics majors start with an incomplete understanding of the physical relationships connecting an infinitesimal source with an infinitesimal space. We do, however, find that middle-division physics students do implement infinitesimal reasoning in many different ways, some correct and others incorrect. In particular, we note that many students make sketches that

[1] R. Knight, Physics for Scientists and Engineers: A Strategic Approach, $3^{\text {rd }}$ Edition (2013).

[2] D. Hu \& S.R. Rebello, in PERC Proceedings (2012).

[3] S. Jones, in RUME Proceedings (2017).

[4] B. Sherin, Cognition and Instruction, 19, 4 (2001). strongly evoke reasoning about small pieces or segments in an electrostatics context requiring integration. A new and key finding is that students who clearly communicate in their sketches a segment understanding of the infinitesimal were more likely to correctly translate $d q$ into $(Q / L) d x$ for use in an integral. This and prior research suggest that it is important for instructors at all levels to help foster a smallsegment understanding of the infinitesimal.

We caution middle-division physics instructors that many of their students still have incomplete understandings of basic calculus concepts as well as of how calculus can be used to describe physical phenomena. We believe these findings are relevant not just for electromagnetism (which was the context of our study), but also for quantum and classical mechanics, both of which frequently involve constructing integrals using small-segment reasoning.

For lower division physics instructors, we recommend stressing the small-segment conceptualization of the infinitesimal in their classes to help their students complete these calculations successfully. We also note that results like ours have spurred us to engage in discussions with colleagues who teach introductory calculus about how the infinitesimal is taught and used in our respective classrooms. We have found these discussions to be particularly valuable (both for ourselves and our colleagues), as they have helped us gain new insight into our students' experiences in related courses.

\section{ACKNOWLEDGEMENTS}

We would like to thank Linn-Benton Community College for providing the first author sabbatical release to address this and other Physics Education Research questions and the Oregon State University Physics Department for encouraging this line of research.

[5] J. W. Creswell, Research Design: Qualitative, Quantitative, and Mixed Methods Approaches, $4^{\text {th }}$ Ed. (SAGE Pub. 2003).

[6] D. J. Griffiths, Introduction to Electrodynamics, $4^{\text {th }}$ Edition (Cambridge University Press, 2017). 